\title{
Photonic integrated technology for multi-wavelength laser emission
}

\author{
CHEN XiangFei ${ }^{1}$, LIU Wen ${ }^{2}$, AN JunMing ${ }^{3}$, LIU Yu ${ }^{3}$, XU Kun ${ }^{4}$, WANG Xin ${ }^{3}$, LIU JianGuo ${ }^{3}$, \\ JI YueFeng ${ }^{4} \&$ ZHU NingHua ${ }^{3 *}$ \\ ${ }^{1}$ National Laboratory of Microstructures, Nanjing University, Nanjing 210093, China; \\ ${ }^{2}$ State Key Laboratory of Optical Communication Technologies and Networks, Wuhan Research Institute of Posts \& Telecommunications, Wuhan \\ 430074, China; \\ ${ }^{3}$ Institute of Semiconductors, Chinese Academy of Sciences, Beijing 100083, China; \\ ${ }^{4}$ Institute of Optical Communication and Optoelectronics, Beijing University of Posts and Telecommunications, Beijing 100876, China
}

Received February 24, 2011; accepted July 15, 2011

\begin{abstract}
We summarized the design, fabrication challenges and important technologies for multi-wavelength laser transmitting photonic integration. Technologies discussed include multi-wavelength laser arrays, monolithic integration and modularizing coupling and packaging. Fabrication technique requirements have significantly declined with the rise of reconstruction-equivalent-chirp and second nanoimprint mask technologies. The monolithic integration problem between active and passive waveguides can be overcome with Butt-joint and InP array waveguide grating technologies. The dynamic characteristics of multi-factors will be simultaneously measured with multi-port analyzing modules. The performance of photonic integration chips is significantly improved with the autoecious factors compensation packaging technique.
\end{abstract}

photonic integration, laser, multi-wavelength

Citation: Chen X F, Liu W, An J M, et al. Photonic integrated technology for multi-wavelength laser emission. Chinese Sci Bull, 2011, 56: 3064-3071, doi: $10.1007 / \mathrm{s} 11434-011-4677-7$

Photonic integrated circuits (PICs) are important for realizing large-capacity and low-energy consumption future optical networks. In PICs, parallel photonic integrated chips with multi-wavelength laser emission will become important for high-speed data transmission, and with largescale photonic integration, will undertake similar tasks to integrated circuits. Thus, PIC has received much recent research attention. Since 2004, the United States, Europe and Japan have launched large-scale research projects involving photonic integration, including the IRIS and LASOR projects in the United States, the EuroPIC, PLATON and HELIOS projects in Europe and the PiFAS project in Ireland. The world pioneer in PIC technology is the Infinera Corporation from the United States. They have developed PIC-based wavelength division multiplexing (WDM) transmission equipment, which is now commercially avail-

*Corresponding author (email: nhzhu@semi.ac.cn) able. The Infinera PIC chip is realized through the integration of more than 50 discrete functions in a single monolithic chip.

The structure of a PIC with multi-wavelength laser transmitter is shown in Figure 1. The chip is constructed of a multi-wavelength laser array, modulator array, light detector array for monitoring optical power, semiconductor optical amplifier (SOA) array for power balancing and WDM combiner (usually an array waveguide grating (AWG)). Although the technology for discrete components is largely mature in commercial applications, it still remains a significant challenge to integrate these different functional optoelectronic devices into a single chip. There are many scientific and technical challenges, including the preparation compatibility issues for different functional micro-nano structures, the multi-wavelength laser emission that meets the requirement of ITU-T specifications, the laser mode stability, and laser mode crosstalk problems caused by the 


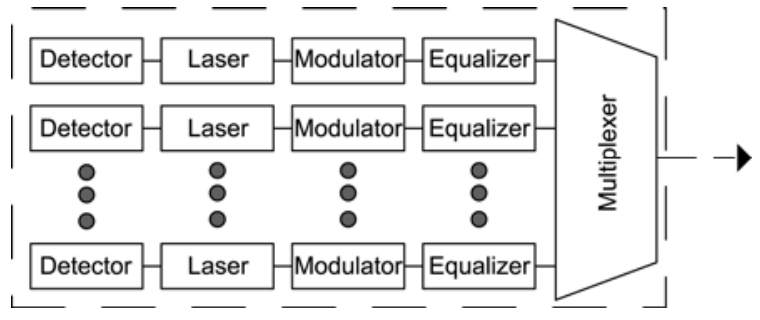

Figure 1 Integrated chip for multi-wavelength laser transmitter.

interaction of microwaves and light waves inside the chip.

Light detector array, SOA array and WDM combination in PICs is well advanced and will not be further discussed here. However, there is a big difference between multiwavelength laser arrays and single-channel lasers, and many important issues need to solve. For example, to achieve accurate $100 \mathrm{GHz}$ wavelength spacing, a grating period spacing of $0.12 \mathrm{~nm}$ is necessary. Traditional holographic exposure to fabricate gratings cannot meet these demanding requirements to achieve the precise control of the lasing wavelength. Point-by-point writing techniques using highprecision electron beam (E-beam) lithography can achieve a precision of $0.1 \mathrm{~nm}$. However, this technology has a long writing time, high cost, low yield and poor reproducibility. E-beam lithography is a useful laboratory tool but unsuitable for large-scale commercial applications [1]. The Infinera Corporation uses selective area growth (SAG) method to control the structural parameters of the active layer of each laser, including thickness and material components. Fine wavelength alignment in the multi-wavelength laser array can be realized through micro-heating resistors to adjust the temperature of individual lasers [2]. However, this method is costly and unable to meet the requirements of low-cost and large-scale production.

To solve these important technology issues for chips with multi-wavelength laser emission, innovative design is necessary. Based on the epitaxial growth of different band structures, low-cost large-scale PIC production can be realized. The present paper discusses the design and manufacturing technologies of multi-wavelength laser arrays, as well as their monolithic integration, coupling and packaging for modules.

\section{Multi-wavelength laser array approaches}

A semiconductor laser diode has two parts, the gain region and laser cavity. The performance of the distributed feedback (DFB) semiconductor laser depends on two factors: the material properties and the grating structure of the laser diode. Two important issues need to be addressed. One is the mechanism for generating multi-wavelength lasing and the other one is the high yield of the single-longitudinal mode (SLM), which is strongly related to the grating structure. Currently, there is no generally accepted solution to provide high performance devices at a low-cost that can be mass produced. New methods are necessary, such as incorporating novel functional microstructures.

\subsection{REC technology}

Reconstruction-equivalent-chirp (REC) technology is compatible with the manufacture of common integrated circuits (ICs). Based on conventional photolithography, it can realize new functional microstructures to simultaneously generate multi-wavelength lasing and achieve high SLM yields. And then the fabrication low-cost to meet the requirement of mass production for multi-wavelength laser arrays (MWLA). REC technology was initially proposed in fiber Bragg gratings [3,4], and has been applied to optical filters [5], fiber lasers [6], OCDMA en/decoders [7] and other important optical devices. The main idea of REC is to use large sampling pattern structures (micron-scale) instead of small grating structures (nano-scale) to achieve the same excellent performance. In general, the scale of microstructures for REC is above $1.5 \mu \mathrm{m}$, which is far greater than $130 \mathrm{~nm}$ (i.e. about half of the grating period of a DFB laser) achievable in conventional methods. Using REC technology, we can produce high-performance low-cost DFB laser arrays based on conventional methods for single-channel DFB lasers, and the fabrication process is similar to that for ordinary DFB lasers with uniform gratings. The only difference between them is that in REC technology, the sampling pattern is formed on the holographic uniform grating through the second photolithography. In REC technology, the lasing wavelength depends on the sampling structure $[8,9]$, thus we can achieve lasers with different lasing wavelengths by designing different sampling structures in the second photolithography. The first holographic exposure and second conventional photolithography are mature production technologies, so the mass production of multi-wavelength DFB semiconductor lasers is possible for conventional fabrication platforms using REC technology. The optical spectrum, P-I characteristics and optical field distribution in the cavity of a DFB laser have been previously reported [10], and it has been concluded that DFB lasers based on REC technology have the same optical characteristics as conventional DFB lasers. In 2009, a monolithic 30-wavelength semiconductor laser array was reported based on REC technology [11], and this demonstration further verified the potential applications of REC technology in laser arrays [12].

REC-based lasers with complex structures have been developed. We have obtained a corrugation pitch modulation (CPM) laser and multi-phase shift laser. These structures can greatly suppress the spatial hole burning effect of the lasers [13-15]. Even at high current, the SLM of these lasers can be maintained and their modulation performance can be significantly improved.

Figure 2 shows an experimental spectrum of a 6-wavelength $\lambda / 4$ phase-shifted DFB laser array based on REC 


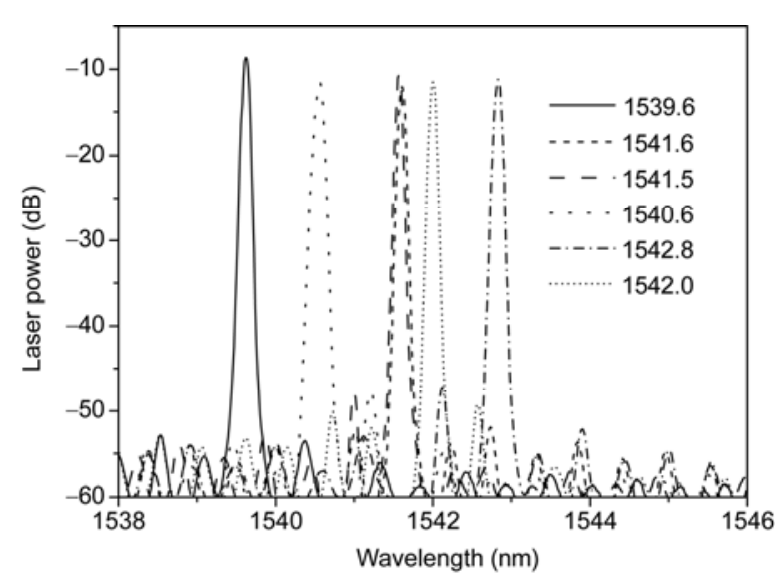

Figure 2 Measured spectrum of a $\lambda / 4$ phase-shifted DFB laser array based on REC technology. The drive current is $40 \mathrm{~mA}$ and the number of the lasers is 6 .

technology. Lasers in the array were measured consecutively. Compared with previous reports, the number of lasers in the array is smaller but the performance of individual lasers is much better. The drive current is $40 \mathrm{~mA}$ and the side mode suppression ratio (SMSR) is in excess of $36 \mathrm{~dB}$, which meets the requirements of the telecom standard $(>35 \mathrm{~dB})$.

The wavelength spacing of a laser array is an important issue for PIC chips and their application to dense wavelength division multiplexing (DWDM) systems. Due to uniformity problems in fabrication and material growth, errors exist in the control of the wavelength spacing. For example, when a laser is made using high-precision E-beam lithography, the random error of the channel spacing is about \pm 1 $\mathrm{nm}[16,17]$, and the maximum error may be in excess of 2 $\mathrm{nm}$. In Figure 2, the design wavelength spacing is $0.4 \mathrm{~nm}$, and the experimental result is shown in Figure 3. The average wavelength spacing is $0.41 \mathrm{~nm}$ with a maximum deviation of $\pm 1 \mathrm{~nm}$, which indicates that the wavelength controlling ability of REC technology is similar to that of the E-beam method. The lithography mask used to form the REC pattern in the experiment is very important for the wavelength control of the DFB laser. The lithography mask used in the experiment in Figure 2 is an ordinary lithography mask and its precision will affect the wavelength accuracy. It is believed that when fabrication is improved and more precise masks are available, better experimental results will be obtainable.

Using high-precision E-beam lithography and good fabrication processes, the minimum deviation in wavelength control of the laser array is about $\pm 1 \mathrm{~nm}$, according to previous reports. This could meet the requirements for coarse wavelength division multiplexing (CWDM) systems, but not for DWDM systems. The Infinera Corporation recently used local temperature control to adjust the laser wavelength, to meet the standard for wavelength spacing in DWDM systems.

The above experimental testing and discussion shows
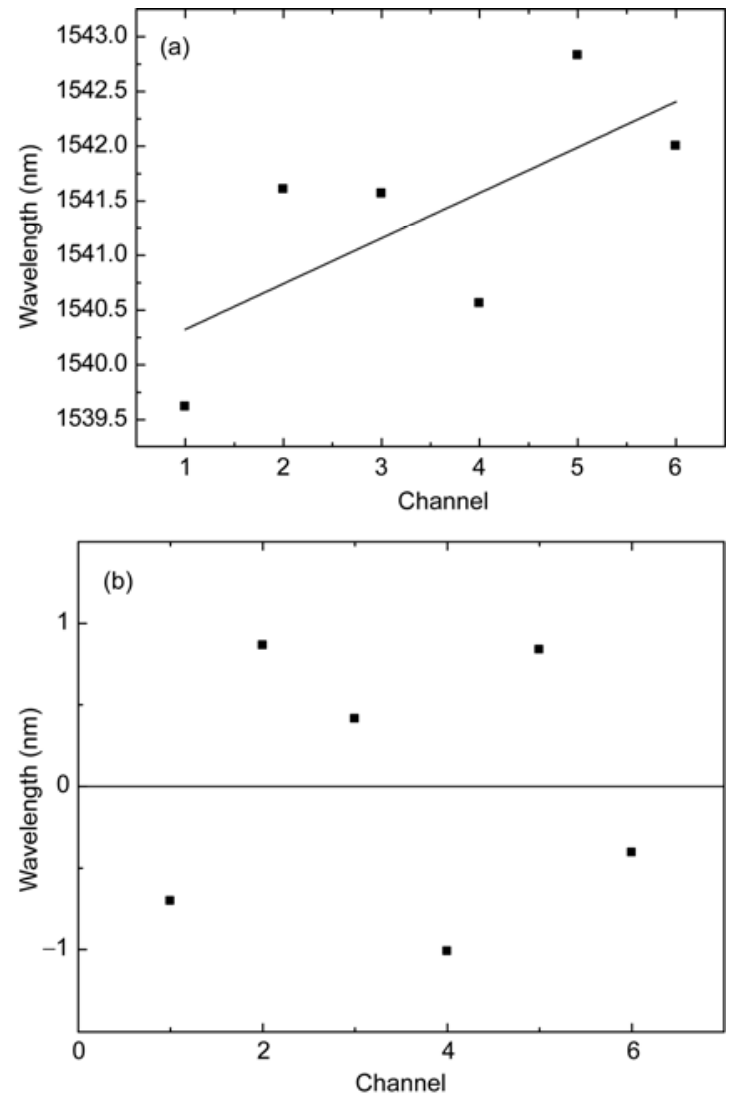

Figure 3 Laser wavelengths of the lasers in the array shown in Figure 2. The drive current is $40 \mathrm{~mA}$ (a) lasing wavelength variation with channels; (b) lasing wavelength error.

that the properties of laser arrays fabricated by REC technology are similar to those using traditional E-beam lithography. This indicates that REC technology can be applied to the fabrication of PICs. In combination with other approaches, REC technology can also further improve the performance of laser arrays, such as using asymmetric sampling structures [18]. With these approaches, REC-based laser arrays will be better optimized, which will enhance the development of large-scale and low-cost laser arrays.

\subsection{Nano-imprint lithography}

Nano-imprint lithography (NIL) technology is a promising high-resolution and low-cost nano-scale duplication technology. The principles of NIL are shown in Figure 4. A patterned stamp is imprinted onto a substrate, usually a very thin polymer film, to achieve pattern transfer. Heating or UV exposure is then used to cure the transferred pattern, and to finish the photonic lithography process. NIL transfers resists through imprinting rather than changing the chemical structure by exposure, thus resolution is not influenced by light diffraction, scattering, optical interference inside the resist, substrate reflection or other factors. Thus, the limitations of traditional photolithographic processes are overcome. NIL does not require complex lenses or light sources, 


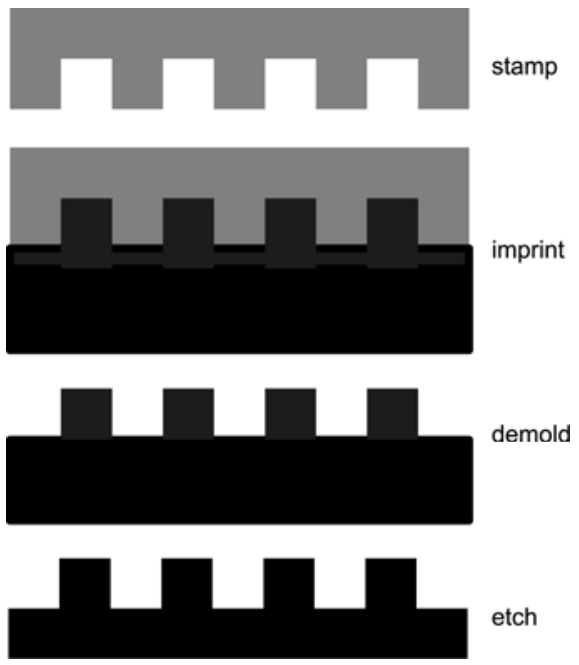

Figure 4 Schematic diagram showing the principles of nano imprint technology.

and can provide repeatable uniform nano-scale structure fabrication in large volumes. This technology is low-cost and suitable for mass production [19-22].

An important technology for fabricating multi-wavelength laser integrated chips using NIL is the fabrication of high quality imprint stamps. The wavelength interval of DFB lasers which meets the technical requirements of WDM is only $0.8 \mathrm{~nm}(100 \mathrm{GHz})$. The corresponding grating period in the C-band is about $246 \mathrm{~nm}$, and the difference in the grating period of adjacent wavelengths is only $0.12 \mathrm{~nm}$. Common E-beam lithography can ensure a high resolution of only $8 \mathrm{~nm}$. The method of gain function is used to overcome difficulties and improve quality. For a given grating size, a scale factor is set which is added to subsequent gratings. We have also developed a secondary template technology that uses E-beam lithography to directly write to a small quartz $10 \mathrm{~mm} \times 10 \mathrm{~mm}$ template, then repeatedly imprints by step-flash to transfer patterns onto 3-inch silicon wafers, using the template as a stamp. The template structure is shown in Figure 5. In each small unit, there are 13 gratings of different wavelength that meet the requirements for DWDM. The topography of the gratings is shown in Figure 6 . The grating period is about $240 \mathrm{~nm}$ after imprinting, and the phase shift is very clear. The grating is uniform over the entire InP epitaxial wafer, and almost no imprinting defects were observed [23].

We have tested the characteristics of multi-wavelength laser chips. From 1540 to $1560 \mathrm{~nm}$, we selected a laser chip every $1.6 \mathrm{~nm}(200 \mathrm{GHz})$ and tested its output spectrum. The spectrum in Figure 7 shows that the laser wavelength is distributed in the C-band. After a TEC temperature control, the wavelength can meet the requirements of ITU-T standard G. 692 for DWDM lasers. SMSR is higher than $40 \mathrm{~dB}$ and the $20 \mathrm{~dB}$ bandwidth is less than $0.2 \mathrm{~nm}$. This result indicates that the characteristics of the DFB laser using NIL are as

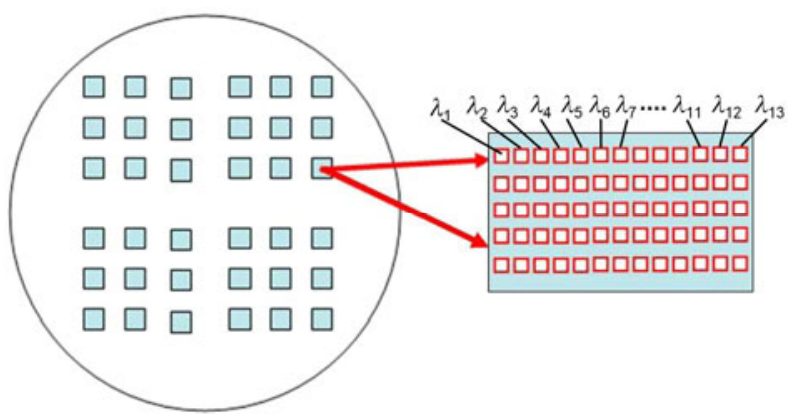

Figure 5 Schematic diagram showing the stamp in the C-band 13-wavelength DFB laser array.
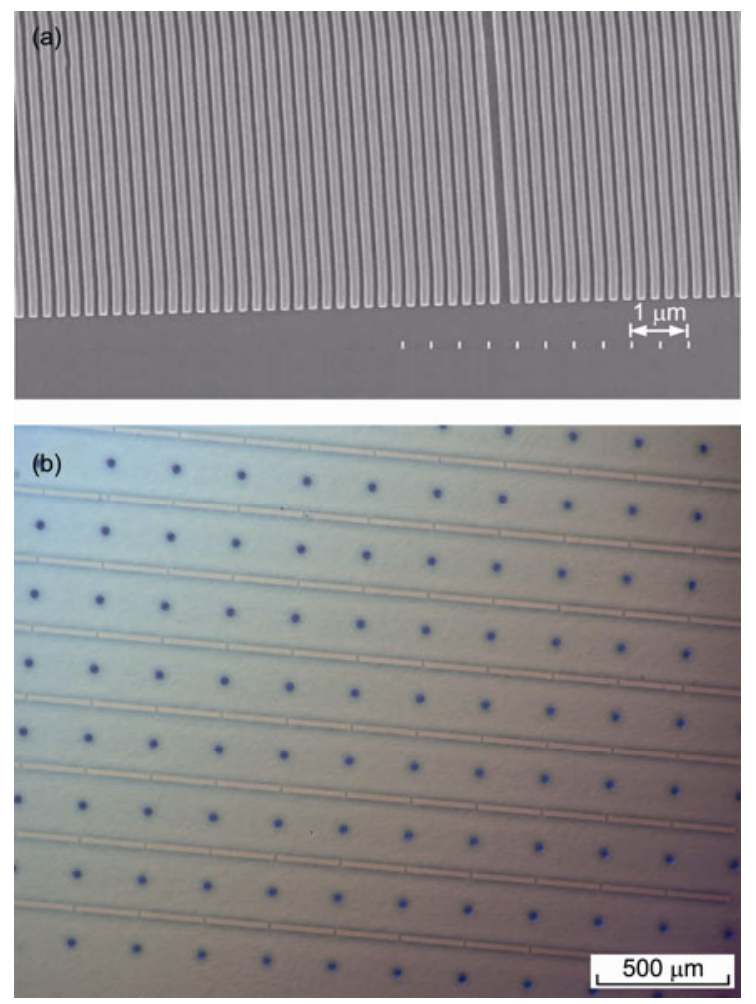

Figure 6 Topography images of the grating fabricated by nanoimprint lithography (a) enlargement of a grating and (b) large area of the grating arrays.

good as those using E-beam lithography. This type of laser can meet the requirements for DWDM systems, and are low-cost and can be mass-produced. According to the ITU-T standard, if we selected a $200 \mathrm{GHz}$ interval and TEC technology to exactly control the wavelength, then for a DWDM system with a $100 \mathrm{GHz}$ interval, the wavelength control precision must be less than $12.5 \mathrm{GHz}$. The corresponding grating control accuracy is also required to be $\pm 0.1 \mathrm{~nm}$. E-beam lithography and NIL cannot provide such initial wavelength accuracy. Even if it could be realized, there is no significance for the requirements of a wide range of operating temperatures. It is necessary to control the 


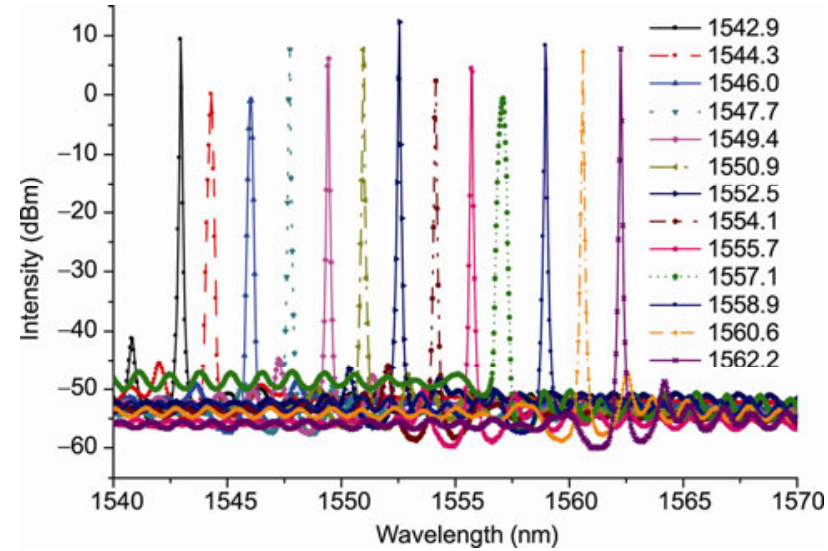

Figure 7 Emission spectral characteristics of nanoimprint multi-wavelength lasers.

initial wavelength in the range of $\pm 100 \mathrm{GHz}$. This is because although we can exactly control the wavelength using TEC, its tuning range is limited. If the chip temperature becomes too high during TEC, the lasers life expectation will be significantly reduced. We have referred to the performance index and design of similar international products. Initial NIL stamps were set at a $200 \mathrm{GHz}$ interval and good results are obtained. There is great potential to control the initial wavelength in the range of $\pm 50 \mathrm{GHz}$, if the process is optimized.

\section{Monolithic technology}

It is important to be able to fabricate reproducible, high quality active-passive waveguide transitions in PIC technology. Active waveguide refers to a guide containing material whose bandgap energy is less than or near to the propagation photon energy. Passive waveguides have a bandgap energy substantially larger than the propagation photon energy, to insure low absorption loss. Multi-wavelengths and multi-signal transition/receiving must be able to be handled in complex InP PICs. Multi-wavelengths are multiplexed to one fiber in a transmitter module, and are then demultiplexed before electro manipulation. As a multiplexer/demultiplexer, InP AWG is an important passive device.

\subsection{Multi-function and microstructure coupling between active and passive portions}

A high efficiency coupling method is the butt-joint between active and passive waveguides with different bandgaps, and different vertical and longitudinal structures. As shown in Figure 8, active waveguides are connected to passive waveguides at the end. Calculation of butt-joint coupling efficiencies is carried out by numerically computing the overlap integral between the two structures [24]. An advantage of

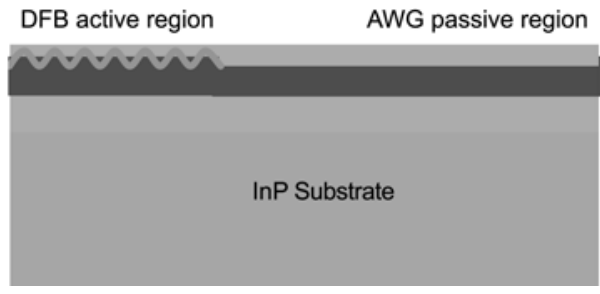

Figure 8 Structure of a butt-joint coupling between a DFB LD and passive waveguides.

this method is the independent selection of compositional and dimensional design parameters for the two waveguides. In principle, the coupling efficiency between the active and passive waveguides is nearly $100 \%$, while reported experimental coupling efficiencies have so far achieved a maximum of $90 \%$. Other waveguide coupling techniques, such as vertical grating coupling, have also been demonstrated. However, the designs simplicity, flexibility and favorable fabrication tolerances makes butt-joint coupling quite an attractive method.

\subsection{Multi-wavelength, low-loss multiplexing technique (InP AWG)}

To avoid polarization dependence and reduce bending radii, a deep ridge InGaAsP/InP waveguide structure can be adopted [25]. The InP buffer layer, InGaAsP core layer with $1.05 \mathrm{Q}$, and InP overcladding layers are grown epitaxially in sequence on a InP semi insulating substrate. The deep ridge waveguide is then etched using induced coupler plasma after lithography, which is shown in Figure 9. The width of the waveguide is determined by waveguide polarization and the InGaAsP bandgap. With larger waveguide width, the waveguides which have low bandgaps can easily achieve polarization independence, and have a good fabrication tolerance. To reduce absorption loss, an undoped or SI-InP overcladding layer is required. The deep ridge waveguide makes the bending radii only $250 \mu \mathrm{m}$, enabling us to fabricate extremely small InP AWG. It has been reported that a 40 channel InP AWG was integrated with 40 EMLs, including 241 discrete function components on a single monolithic InP chip [26]. Figure 10 shows a 10 channel $200 \mathrm{GHz} \mathrm{InP}$

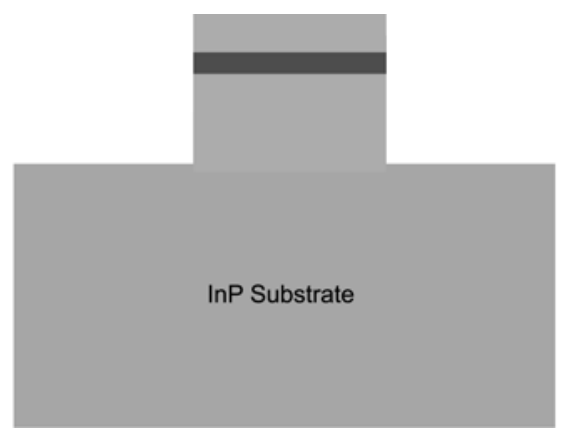

Figure 9 Deep-ridge waveguide with polarization independence. 


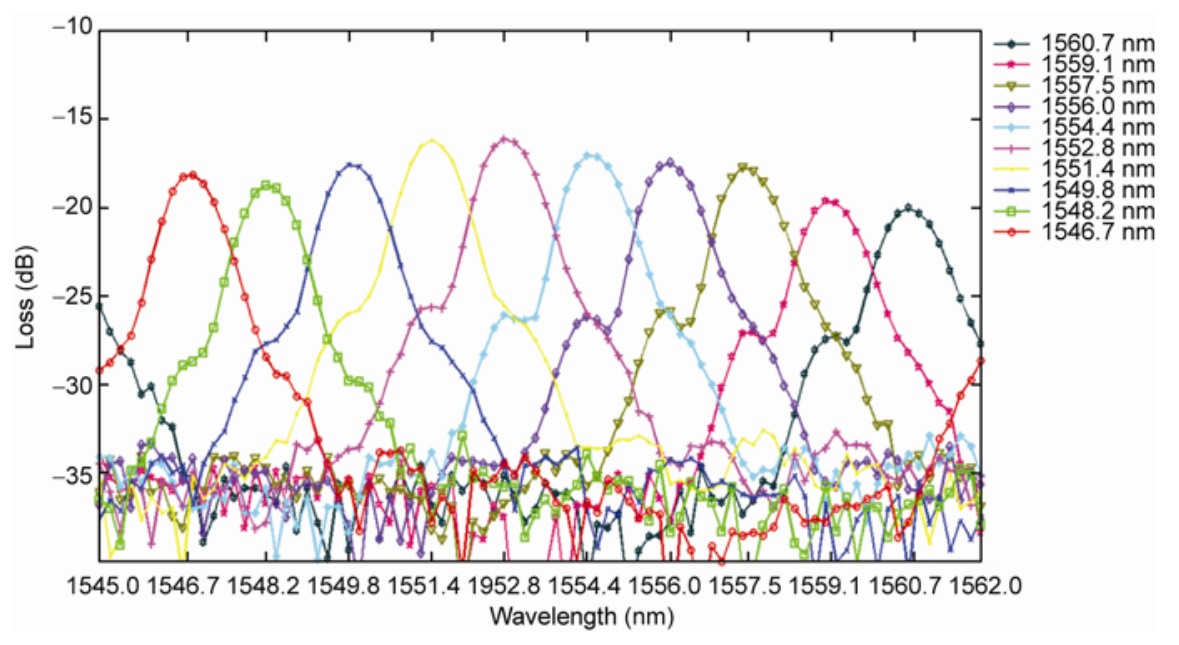

Figure 10 Spectral response of a 10 channels $200 \mathrm{GHz}$ InP AWG.

AWG output spectrum fabricated in our laboratory. The chip dimensions are $4 \times 4 \mathrm{~mm}^{2}$, and the insertion loss is about $-16 \mathrm{~dB}$ at the center output port. The crosstalk is less than $-17 \mathrm{~dB}$.

\section{Measurement analysis and module packaging techniques}

The optoelectronic device is an integral module, in which the energy between microwaves and light waves exchange. To realize the coupling and packaging of the integrated laser chip, many factors such as chip material, electrode structure, packaging circuit and coupling structure must be considered.

\subsection{Measurement analysis of multi-channel lasers}

Under large scale integration conditions, internal coupling and crosstalk between different modes usually occurs in a laser array. In this case, non-linearity such as four-wave mixing becomes apparent, which will affect the multi-wavelength parallel signal and deteriorate the dynamic range, sensitivity, and bit error rate of the system.

The measurement and analysis of the multi-channel laser is based on intrinsic parameters: extraction of the laser and model establishment of the internal light transmission and energy conversion. We have developed two methods for parameter extraction to date. One is to ensure the modulated laser beams inject into the laser under test conditions to obtain the intrinsic response [27]. The other one is based on the deduction of the parasitic parameters in the equivalent circuit model, from which the intrinsic response would be directly obtained [28].

An important difference between a laser array and a separated laser is the coupling and energy exchange of light waves and microwaves between different lasers. To study the cross-modulation and the crosstalk characteristics in an integrated chip array, measurement of multi-port tests [29] and integration models [30], combined with time domain reflectometry (TDR) for signal integrity analysis are used. They have proven effective in our laboratory.

\subsection{Low-reflection optical coupling}

In optical coupling systems, light reflection will affect the stability of the output wavelength and cause additional microwave modulation. Therefore, attention should be given to improving optical coupling efficiency and minimizing the external light reflection [31]. Currently, combined lenses and fiber micro-lenses have been widely used [32]. The former have a large tolerance, and optical alignment is much easier. Adding an optical isolator between the two lenses can reduce the light reflection. The latter is to fabricate the plane or lenses at the fiber end. This has many advantages such as its compact structure, simplicity and low cost. We use a seven degree tilt structure in the output waveguide, with coated lenses, isolator and taper fiber. This optical coupling technique is highly efficient and possesses low reflection [33].

\subsection{Module packaging}

The high frequency characteristics of an integrated optical transmitter module depend on the intrinsic response of the laser or modulator chip, and also on the parasitic parameters in chip electrodes and module packaging. Electro-absorption modulators commonly use P, N coplanar electrodes [34]. The corresponding pad electrodes are fabricated on aluminum nitride materials, which have good thermal conductivity. By flip chip bonding processes or metal bonding technology, multiple bonding wires can be avoided so that low-loss transmission of modulation signal can be achieved. Pack- 
aging parasitic parameters are predominantly introduced from inductance and capacitance, which are caused by bonding wires and electrode pads, respectively. By optimizing the design of bonding wires and pads, we have implemented the compensation of chip parasitic parameters [35].

\section{Conclusions}

Multi-wavelength PIC technology was discussed in this report. A brief description of our own intellectual property technologies in the area of multi-wavelength laser emission and active/passive multifunctional structure integration was presented. For example, based on REC and nano-imprint secondary template technologies, the difficulty of chip preparation can be greatly reduced and thus low-cost fabrication, such as holographic exposure, can be applied for multiwavelength laser arrays. These will be beneficial for mass production. The problem of monolithically integrating active and passive waveguides can be overcome by using end butt joint coupling technology. We established a multi-port model, and based on the dynamic analysis and experimental validation of the multi-wavelength laser, an optimal design of material growth, device fabrication and packaging is achieved and system performance verification can be realized. These core technologies are still in the process of development and refinement, but preliminary experiments show their unique technical advantages. Such technologies support each other, and when combined together it will form a technology for the mass production of high-speed PIC chips. This will provide a solid foundation for the research and application of these chips, which are represented by multi-wavelength laser emission chips.

This work was supported by the National High-Tech Research \& Development Program of China (2011AA0103).

1 Ishii H, Kasaya K, Oohashi H, et al. Widely wavelength-tunable DFB laser array integrated with funnel combiner. IEEE J Sel Top Quantum Electron, 2007, 13: 1089-1094

2 Kish J, Fred A J, Charles H W, et al. Method of operating an array of laser sources integrated in a monolithic chip or in a photonic integrated circuit(PIC). US Patent, US7079720B2, 2002-07-18

3 Chen X F, Luo Y, Fan C C, et al. Analytical expression of sampled Bragg gratings with chirp in the sampling period and its application in dispersion management design in a WDM system. IEEE Photonics Technol Lett, 2000, 12: 1013-1015

4 Dai Y T, Chen X F, Xia L, et al. A realization of arbitrary goal response optical fiber Bragg grating. PRC Patent, CN1560656, 200501-05

5 Dai Y T, Chen X F, Xia L, et al. Sampled Bragg grating with desired response in one channel by use of a reconstruction algorithm and equivalent chirp. Opt Lett, 2004, 29: 1333-1335

6 Jiang D J, Chen X F, Dai Y T, et al. A novel distributed feedback fiber laser based on equivalent phase shift. IEEE Photonics Technol Lett, 2004, 16: 2598-2600

7 Dai Y T, Chen X F, Sun J, et al. High-performance, high-chip-count optical code division multiple access encoders-decoders based on a reconstruction-equivalent-chirp technique. Opt Lett, 2006, 31: 16181620

8 Chen X F. The method and device for fabricating semiconductor lasers based on reconstruction equivalent chirp technology. PRC Patent, CN101034788, 2007-09-12

9 Dai Y T, Chen X F. DFB semiconductor lasers based on reconstruction-equivalent-chirp technology. Opt Express, 2007, 15: 2348-2353

10 Dai Y T, Yao J P. Numerical study of a DFB semiconductor laser and laser array with chirped structure based on the equivalent chirp technology. IEEE J Quantum Electron, 2008, 44: 938-945

11 Li J S, Wang H, Chen X F, et al. Experimental demonstration of distributed feedback semiconductor lasers based on reconstructionequivalent-chirp technology. Opt Express, 2009, 17: 5240-5245

12 Li J S, Chen X F, Zhou N, et al. Monolithically integrated 30wavelength DFB laser array. In: He J J, Duan G H, Koyama F, et al., eds. Proceedings of SPIE-OSA-IEEE Asia Communications and Photonics, SPIE 7631 763104, 2009 Nov25-29, Shanghai

13 Li S M, Shi Y C, Li J S, et al. Experimental demonstration of the corrugation pitch modulated DFB semiconductor laser based on the reconstruction-equivalent-chirp technology. In: Koyama F, Chuang S L, Duan G H, et al., eds. Proceedings of SPIE-OSA-IEEE Asia Communications and Photonics, SPIE 7987, 798704, 2010 Dec9-12, Shanghai

14 Shi Y C, Tu X H, Li S M, et al. Numerical study of three phase shift and dual corrugation pitch modulated DFB semiconductor lasers based on reconstruction equivalent chirp technology. Chinese Sci Bull, 2010, 55: 1944-1950

15 Shi Y C, Li S M, Lu L L, et al. Multiple phase shifts DFB semiconductor laser based on reconstruction equivalent chirp technology. In: Zhu N H, Li J, Amzajerdian F, et al., eds. Proceedings of SPIE 7844 784418, 2010 Nov18-20, Beijing

16 Huang Y D, Sato K, Okuda T, et al. Low-chirp and external optical feedback resistant characteristics in $\lambda / 8$ phase-shifted distributedfeedback laser diodes under direct modulation. IEEE J Quantum Electron, 2002, 38: 1479-1484

17 Li J S, Cheng Y, Yin Z W, et al. A multiexposure technology for sampled Bragg gratings and its applications in dual-wavelength lasing generation and OCDMA en/decoding. IEEE Photonics Technol Lett, 2009, 21: 1639-1641

18 Zhou Y T, Shi Y C, Li S M, et al. Asymmetrical sampling structure to improve the single-longitudinal-mode property based on reconstruction-equivalent-chirp technology. Opt Lett, 2010, 35: 3123-3125

19 Chou S Y, Krauss P R, Renstrom P J, et al. Imprint of sub-25 nm vias and trenches in polymer. Appl Phys Lett, 1995, 67: 3114-3116

20 Chou S Y, Krauss P R, Zhang W, et al. Sub-10 nm imprint lithography and applications. J Vac Sci Technol B, 1997, 15: 2897-2904

21 Hong P S, Lee H H. Pattern uniformity control in room-temperature imprint lithography. Appl Phys Lett, 2003, 83: 2441-2443

22 Hua F, Gaur A, Sun Y, et al. Processing dependent behavior of soft imprint lithography on the 1-10-nm scale. IEEE Trans nanotechnol, 2006, 5: 301-308

23 Liu W, Wang D L, Zhou N, et al. Second imprint template and the fabrication method for second imprint template for nano imprinting. PRC Patent, CN101446759, 2009-06-03

24 Koch T L, Koren U. Semiconductor photonicintegrated circuits, IEEE J Quantum Electron, 1991, 27: 641-653

25 Yuzo, Semiconductor arrayed waveguide gratings for photonic integrated devices. IEEE J Sel Top Quantum Electron, 2002, 8: 11021114

26 Welch D F, Kish F A, Nagarajan R. The realization of large-scale photonic integrated circuits and the associated impact on fiber-optic communication systems. J Lightwave Technol, 2006, 24: 4674-4683

27 Zhu N H, Chen C, Pun E Y B, et al. Extraction of intrinsic response from S-parameters of laser diodes. IEEE Photonics Technol Lett, 2005, 17: 744-746

28 Zhang S J, Zhu N H, Liu Y, et al. Potential frequency bandwidth estimation of TO packaging techniques for photodiode modules. Opt Quantum Electron, 2006, 38: 675-682 
29 Zhang Y, Silvonen K, Zhu N H. Measurement of a reciprocal four-port transmission line structure using the 16-term error model. Microw Opt Technol Lett, 2007, 49: 1511-1515

30 Zhu N H, Hou G H, Huang H P, et al. Electrical and optical coupling in an electro-absorption modulator integrated with a DFB laser. IEEE J Quantum Electron, 2007, 43: 535-544

31 Gnitabourk Y. Effect of relatively strong light injection on the chirp-to-power ratio and 3-dB bandwidth of directly modulated semiconductor lasers. J Lightwave Technol, 1996, 14: 2367-2373

32 Nakagawa G, Kai Y, Yoshida S, et al. Novel optical coupling technique for enhancing the performance of integrated 8-input/1-output SOA gate-switch module. J Lightwave Technol, 2009, 27: 4989-4994

33 Wang X, Xie L, Yuan H Q, et al. Butterfly package devices for semiconductor lasers. PRC Patent, CN1937336, 2007-03-28

34 Tsuzuki K, Kawaguchi Y, Kondo S, et al. Four-channel arrayed polarization independent EA modulator with an IPF carrier operating at $10 \mathrm{~Gb} / \mathrm{s}$. IEEE Photonics Technol Lett, 2000, 12: 281-283

35 Zhu N H, Liu Y, Zhang S J, et al. Bonding-wire compensation effect on the packaging parasitics of optoelectronic devices. Microw Opt Technol Lett, 2006, 48: 76-79

Open Access This article is distributed under the terms of the Creative Commons Attribution License which permits any use, distribution, and reproduction in any medium, provided the original author(s) and source are credited. 